engaged with the posts relating to the topics that scored lowest in the pre-intervention assessment. The question that scored the lowest pre-intervention showed the most engagement on Facebook and biggest increase in score.

Conclusion Posting health related information to a Facebook group is not a replacement for face to face education but may serve as an adjunct to good diabetes care.

\section{G423(P) UNCOMMON PRESENTATION OF SEPTO-OPTIC DYSPLASIA}

A Jeyaraman, C Brew, V Mathew, S Gupta. Paediatrics, Hull Royal Infirmary, Kingston upon Hull, UK

\subsection{6/archdischild-2020-rcpch.365}

Introduction Neonatal metabolic acidosis is a common presentation of wide range of causes varying from sepsis to rare metabolic and endocrine problems. Metabolic acidosis with normal anion gap help as to narrow down the cause as GI loss, RTA or adrenal insufficiency. We present a rare case of neonatal metabolic acidosis turned out to be due to adrenal insufficiency and subsequently diagnosed as SOD. Septo-optic dysplasia (SOD) is a rare congenital malformation syndrome characterised by a triad of optic nerve hypoplasia, midbrain abnormality and pituitary dysfunction.

Case Report 19 day-old girl baby presented with lethargy, poor feeding and poor weight gain. On examination, she was hypothermic, hypoglycaemic, hyperkalaemia with early shock and metabolic acidosis. She was treated as presumed sepsis with dextrose, fluid bolus and antibiotics. She had persistent metabolic acidosis with normal anion gap despite on full feeds. Her septic screening and initial metabolic screening were negative. She was noted to have adrenal insufficiency (cortisol <50), which was confirmed by synacthen test. Her cranial ultrasound was normal. She was sent home with steroid supplements. She was noticed as not fixing and following with roving eye movements at the age of 4 months. Found to have bilateral optic nerve dysplasia and later registered as blind. Further imaging of MRI head confirmed SOD with underdevelopment of optic nerve and hypoplasia of pituitary gland. She failed the dynamic growth hormone stimulation test. In view of her slow growth and low IGF, she was started on growth hormone treatment. She also has hypothyroidism on thyroxin supplements. She is making good progress in her growth and development with hormone supplements and MDT management.

Conclusion Clinical followed by hormonal evaluation and imaging can guide us to its appropriate diagnosis and treatment of SOD. Early recognition of SOD is paramount in order to reduce morbidity and to optimise management. Without adequate treatment, panhypopituitism can have devastating effects on growth and neurodevelopment of children.

\section{G424(P) RE-INTERPRETING THE APPROPRIATE GROWTH HORMONE RESPONSE TO HYPOGLYCAEMIA}

${ }^{1}$ UA Khan, ${ }^{1} \mathrm{FY}$ Wong, 'S Azam, ${ }^{2} \mathrm{O}$ Blake, ${ }^{1,3} \mathrm{AM}$ Murphy, ${ }^{1,3} \mathrm{OM}$ Neylon, ${ }^{1,3} \mathrm{CS}$ O'Gorman. ${ }^{1}$ Dept of Paediatrics, University Hospital, Limerick, Ireland; ${ }^{2}$ Dept of Biochemistry, University Hospital, Limerick, Ireland; ${ }^{3}$ Dept of Paediatrics, Graduate Entry Medical School, University of Limerick, Limerick, Ireland

10.1136/archdischild-2020-rcpch.366
Aims 'Critical samples' consist of assessment of an individual's metabolic and endocrine status during a hypoglycaemia episode to evaluate for a pathological contributor towards aetiology of the episode. Growth hormone $(\mathrm{GH})$ level is part of this work up to assess likelihood of GH deficiency. The aim of this study was to identify if the preset cut off value for growth hormone ' 7 microgram/Litre' during hypoglycaemia is appropriate and how likely values below this are to be indicative of growth hormone deficiency.

Methods The study population consisted of paediatric patients aged up to 16 years old who had critical samples sent during hypoglycaemia over a 12 month period from 1st Jan 2018 until 30th Dec 2018 inclusive at University Hospital Limerick. Retrospective review from a prospectively collected database of glucose and GH levels as well as other growth parameters.

Results Forty blood samples were collected during the study period during hypoglycaemia. One sample was insufficient for analysis of GH level and of the remaining 39, only three (7.6\%) patients exhibited a 'normal' GH response to hypoglycaemia of $\geq 7 \mathrm{mcg} / \mathrm{L}$ and thirty-six patients (92\%) had suboptimal GH levels. Data was skewed to the right with median GH reading of $3.19 \mathrm{mcg} / \mathrm{L}$. The available data on the other parameters i.e. Insulin-like Growth Factor (IGF)-1 levels, Growth Velocity/Height centiles and Growth hormone stimulation test (GHST) was limited but normal.

Conclusion No patient had documented GH deficiency or loss of height centiles, despite only $7.6 \%$ (3/39) having 'adequate' GH response during hypoglycaemia. These data suggest that the optimal level for GH during hypoglycaemia should be reevaluated in larger prospective studies, to reduce unnecessary evaluation for GH deficiency with attendant anxiety and cost generated therein.

\section{G425(P) AVERAGE HBA1C LEVELS IN DIABETIC CHILDREN WITH INSULIN PUMPS AND MULTIPLE DOSE INSULIN}

L Horne, Y Galal, R Wilson. Paediatrics, Blackpool Teaching Hospital NHS Trust, Blackpool, UK

\subsection{6/archdischild-2020-rcpch.367}

Aims To correlate patient's average HbA1c scores to the modality of insulin therapy utilised to identify whether there is a statistically significant difference in the average HbA1c levels of patients who initially received multiple dose insulin (MDI) and then were commenced on continuous subcutaneous insulin infusions (CSCI). This information would then be used to deliver realistic counselling for patients changing from MDI to CSCI based on real data from our trust.

Methods Within this service evaluation, average HbA1c scores were calculated for all paediatric patients who were initially taking multiple dose insulin therapy and then subsequently commenced subcutaneous insulin infusions. The average HbA1c scores were reviewed at 1 year prior to CSCI and then 1 year and 2 years after the change in modality of insulin delivery. The service evaluation was conducted in 2017 and then again in 2019.

Results In 2017, a mean difference of in HbA1c of 2.03 (95\% confidence interval $1.17-2.88$ ) was seen with a significant difference of 0.033 . This concludes that there was no significant difference between the average $\mathrm{HbA1c}$ of patients 
on MDI and their average HbA1c values when on CSCI a year later. When repeated in 2019, there was an average improvement in HbA1c levels of $48 \%$ and at 24 months an average of $53 \%$ improvement.

Conclusion This data is utilised to demonstrate to staff and patients how CSCI alone is no guarantee for normalising poorly controlled HbA1C levels. We must continue to take a holistic approach to the counselling and management of our type 1 diabetes paediatric patients.

\section{G426(P) DELAYED OR ABSENT? USE OF NEXT GENERATION SEQUENCING DIAGNOSTIC TOOLS IN A UK PUBERTY COHORT}

HR Patel, SR Howard, T Saengkaew, L Dunkel. Endocrinology, William Harvey Research Institute, London, UK

\subsection{6/archdischild-2020-rcpch.368}

Background and Aim Isolated delayed puberty (DP) and congenital hypogonadotropic hypogonadism $(\mathrm{cHH})$ are causes of severe delayed puberty that are almost clinically indistinguishable. Treatment is condition-specific and time-sensitive, yet existing methods to differentiate the two lack sensitivity and specificity. Genetic testing has been proposed as a potentially accurate diagnostic investigation. This project aimed to broaden our understanding of the genetic basis of severe delayed puberty to aid clinical diagnosis and prognosis of individual patients.

Methods Phenotype and genotype data of a UK cohort of 70 (probands $=24$, relatives $=46$ ) was studied. Whole exome sequencing was performed on probands and variants were filtered to identify candidate genes. Sanger sequencing was used to confirm variants and assess segregation with trait in pedigrees. Published literature, bioinformatics databases and prediction software tools were used to identify likely deleterious variants.

Results Following whole exome sequencing of 24 probands, 27 variants were identified in 21 candidate genes. 10 of these variants were found to be likely deleterious by prediction tools and 1 was proven to be definitively deleterious by previously published literature. 5 candidate genes had previously identified variants associated with $\mathrm{cHH}, 1$ candidate gene had variants previously associated with isolated DP and another candidate gene had variants previously associated with both phenotypes. Clinical diagnoses was established in $46 \%$ of probands. After genetic testing, a further 38\% had a likely pathogenic variant consistent with a diagnoses of $\mathrm{cHH}$ or isolated DP.

Conclusion A likely genetic diagnoses was established in 69\% of probands with previously unclassified severe delayed puberty. Thus, identification of gene mutations known to cause $\mathrm{cHH}$ or isolated DP helps to clarify diagnoses. Genetic testing has the potential to allow time-sensitive, condition-specific treatment and future planning for individual patients. Though clinical significance of variants remains uncertain, SEMA6A, SEMA3E and HS6ST1 were amongst candidate genes highlighted as potentially pathogenic providing plenty of scope for future study.

\section{G427(P) ABSTRACT WITHDRAWN}

\section{G428(P) THE 50 MOST INFLUENTIAL STUDIES ON PAEDIATRIC DIABETIC KETOACIDOSIS: A BIBLIOMETRIC ANALYSIS}

M Nandakumar, MM Maturana, E Vouzonis. Department of Paediatrics, North Middlesex University Hospital, London, UK

\subsection{6/archdischild-2020-rcpch.369}

Objectives Diabetic ketoacidosis (DKA) in paediatrics is a common condition and often the first presentation of type 1 diabetes mellitus in children. The number of citations a study receives can be used as a marker of academic influence, and can help to identify strong and weak areas of research within a particular field. We aimed to identify the 50 most influential studies relating to paediatric DKA by citation number, and analyse their characteristics.

Methods The Web of Science database was used to determine the studies that were most frequently cited. We searched for studies that included 'Diabetic ketoacidosis' or 'DKA' along with 'paediatric' or 'children' or 'child' or 'infant' in the title. Data extracted included publication year, article type and focus, publication journal, institution and country, level of evidence (LOE) and total citation number. Published guidelines were excluded from the LOE rankings. Citation density was also calculated as number of citations per year since publication.

Results Our search returned 410 studies. The top 50 studies were published between 1976 to 2014. The most common study topics were epidemiology and pathophysiology, with many studies relating to cerebral oedema $(n=19)$. The mean number of citations was 74.7 (standard deviation 60.3, range 28-375). These were published in 21 journals, led by Pediatric Diabetes $(\mathrm{n}=9)$. They originated from 9 different countries, with institutions from the United States of America publishing the majority $(\mathrm{n}=30)$, and the University of California, Davis publishing the most studies $(n=7)$. One RCT was identified, 39 non-randomised clinics studies were included. The remainder included reviews, basic science studies and guidelines. 10 studies were classified as LOE 1, and 11 studies as LOE 4.

Conclusions The 50 top cited studies on paediatric DKA relate mostly to epidemiology and pathophysiology. Cerebral oedema in paediatric DKA patients has received increased attention by researchers and institutions. LOE was low, with only one RCT identified. Though these studies are the most influential in the field, perhaps the evidence base for this field would benefit from further high quality trials.

\section{G429(P) DIAGNOSIS OF DIABETES MELLITUS IN CHILDREN, INCLUDING FACTORS LEADING TO DELAYED DIAGNOSIS}

M Thomas, A Ali. Paediatrics Department, Northwick Park Hospital, London, UK

\subsection{6/archdischild-2020-rcpch.370}

Aims To identify patient demographics, presentation and contributing factors towards delay in new diagnosis of diabetes mellitus in children, and to compare this to results from a similar audit in 2013.

Methods A retrospective audit of electronic records of all patients identified from the local paediatrics diabetes caseload database with new diagnoses between 1st January and 31st December 2018. Inclusion criteria included age at diagnosis 